\title{
Characterizing Matchings as the Intersection of Matroids*
}

\author{
Sándor P. Fekete ${ }^{\dagger} \quad$ Robert T. Firla Bianca Spille $^{\dagger}$
}

\begin{abstract}
This paper deals with the problem of representing the matching independence system in a graph as the intersection of finitely many matroids. After characterizing the graphs for which the matching independence system is the intersection of two matroids, we study the function $\mu(G)$, which is the minimum number of matroids that need to be intersected in order to obtain the set of matchings on a graph $G$, and examine the maximal value, $\mu(n)$, for graphs with $n$ vertices. We describe an integer programming formulation for deciding whether $\mu(G) \leq k$. Using combinatorial arguments, we prove that $\mu(n) \in \Omega(\log \log n)$. On the other hand, we establish that $\mu(n) \in O(\log n / \log \log n)$. Finally, we prove that $\mu(n)=4$ for $n=5, \ldots, 12$, and sketch a proof of $\mu(n)=5$ for $n=13,14,15$.
\end{abstract}

Keywords: matching, matroid intersection

AMS classification: 05B35, 05C $70,90 \mathrm{C} 27$

\section{Introduction}

Many combinatorial optimization problems can be viewed as optimization problems over independence systems. Some of them are polynomially solvable, e.g., spanning trees in graphs, the branching problem in digraphs, or the matching problem. Others are known to be NP-complete, like the traveling salesman problem (TSP) or the stable set problem (cf. 1, 7] 8]). Among the problems with a polynomial-time algorithm, the matching problem is generally recognized as one of the "hardest", and the famous blossom algorithm by Edmonds 2 is one of the highlights of combinatorial optimization.

Another seminal result on the optimization in independence systems was also given by Edmonds [3, who proved that the optimization problem over the intersection of two matroids is solvable in polynomial time. Algorithms for this

\footnotetext{
*An earlier version appears as an extended abstract in the Proceedings of COMB'01 [5].

${ }^{\dagger}$ Abteilung für Mathematische Optimierung, TU Braunschweig, s.fekete@tu-bs.de

${ }^{\ddagger}$ Institut für Mathematische Optimierung, Otto-von-Guericke-Universität Magdeburg, [firla, spille] @imo.math. uni-magdeburg. de. Supported by the "Gerhard-Hess-Forschungsförderpreis" (WE 1462) of the German Science Foundation (DFG) awarded to R. Weismantel.
} 
problem were given by Edmonds [4, Frank [6], and Lawler [11, 12. Unfortunately, this cannot be generalized to the case of three or more matroids: As the NP-complete TSP can be written as an optimization problem over the intersection of three matroids, it is highly unlikely that a polynomial-time algorithm exists.

In this paper, we consider a problem that combines aspects of both problems that were solved by Edmonds: How many matroids need to be intersected to characterize the matchings of a graph? This problem is somewhat related to work by Jenkyns [9] and Korte and Hausmann [10, who described approximation guarantees for the greedy algorithm; these guarantees depend on the rank quotient, which depends on the number of matroids needed for characterizing the independence system. Clearly, we are not primarily interested in these approximation guarantees for matching. However, we believe that the problem of describing an independence system as the intersection of few matroids is an interesting combinatorial problem in its own right.

The rest of this paper is organized as follows. After some technical preliminaries in Section 22 Section 3 gives a number of general structural results. In particular, we give a precise characterizations of graphs for which the set of matchings can be represented as the intersection of two matroids, and an Integer Programming formulation for the problem of minimizing the number of matroids that are necessary for representing the matchings of an input graph. In Section 4 we prove that $K_{n}$ needs at least $\Omega(\log \log n)$ matroids, while Section 5 establishes an upper bound of $O(\log n / \log \log n)$ for the number of matroids needed for any graph with $n$ vertices. The final Section [ 6 describes the actual values of matroids that are necessary for graphs with up to 15 vertices.

\section{Preliminaries}

Let $S$ be a finite set and $\mathcal{I}$ be a family of subsets of $S$. $\mathcal{I}$ is an independence system on $S$ if $\varnothing \in \mathcal{I}$ and if $J^{\prime} \subseteq J$ and $J \in \mathcal{I}$ then $J^{\prime} \in \mathcal{I}$. The subsets of $S$ belonging to $\mathcal{I}$ are called independent, otherwise dependent. The minimal dependent subsets of $S$ are the circuits of $\mathcal{I}$. The circuit system $\mathcal{C}$ of $\mathcal{I}$ is the set of circuits of $\mathcal{I}$ and $\mathcal{I}=\{J \subseteq S: C \nsubseteq J$ for all $C \in \mathcal{C}\}$. A maximal independent subset of a set $A \subseteq S$ is a basis of $A$. An independence system $\mathcal{I}$ on $S$ is a matroid if for every subset $A \subseteq S$ all its bases have the same cardinality. For further background, see Oxley 13 and Welsh 14. Here we just state another useful fact.

Proposition 1 The circuit system $\mathcal{C}$ of an independence system $\mathcal{I}$ is the circuit system of a matroid if and only if for all $C_{1} \neq C_{2} \in \mathcal{C}$ with $C_{1} \cap C_{2} \neq \varnothing$ and for all $c \in C_{1} \cap C_{2}$ there exists $C \in \mathcal{C}$ such that $C \subseteq C_{1} \cup C_{2} \backslash\{c\}$.

Any independence system is the intersection of finitely many matroids: Let $\mathcal{C}$ be the set of circuits of $\mathcal{I}$. For $C \in \mathcal{C}$, let $\mathcal{M}_{C}$ be the matroid on $S$ with circuit system $\{C\}$, i.e., $\mathcal{M}_{C}=\{J \subseteq S: C \nsubseteq J\}$. Then $\mathcal{I}=\bigcap\left\{\mathcal{M}_{C}: C \in \mathcal{C}\right\}$. This, however, may not be the most economical way to describe $\mathcal{I}$, because we may 
be able to cover several circuits by the same matroid. In the following, we write $\mu(\mathcal{I})$ for the minimum number of matroids necessary for this task. Throughout the rest of this paper, $\mathcal{I}$ is the set of matchings of a graph, which we describe in the following.

Consider a finite graph $G=(V, E)$. A matching in $G$ is a set of edges that are pairwise disjoint. The set $M(G)$ of matchings in $G$ forms an independence system on $E$. For simplicity, we write $\mu(G)$ for $\mu(M(G))$, i.e.,

$$
\mu(G)=\min \{m \in \mathbb{N}: M(G) \text { is the intersection of } m \text { matroids }\} .
$$

Furthermore, $\mu(n)$ is used for the maximum $\mu(G)$ on graphs with $|V| \leq n$, i.e.

$$
\mu(n)=\max \{\mu(G):|V| \leq n\} .
$$

It is easy to see that the circuits of $M(G)$ are the sets that consist of two intersecting edges. (The reader should keep in mind that throughout the rest of this paper, the term circuit refers to such a pair of edges.) We call a circuit an $i$-circuit if its edges intersect in vertex $i$. We denote the circuit $\{i j, i k\}$ with the two edges $i j$ and $i k$ by $i^{j k}$.

\section{General Characterizations}

The following easy lemma implies that $\mu(n)=\mu\left(K_{n}\right)$.

Lemma 2 Let $G^{\prime}=\left(V^{\prime}, E^{\prime}\right)$ be a subgraph of $G=(V, E)$. Then $\mu\left(G^{\prime}\right) \leq \mu(G)$.

Proof: Let $M(G)$ be the intersection of matroids $\mathcal{M}_{1}, \ldots, \mathcal{M}_{m}$ on $E$. For $a=1, \ldots, m$, let $\mathcal{M}_{a}^{\prime}:=\left\{J: J \subseteq E^{\prime}, J \in \mathcal{M}_{a}\right\}$ be the restriction of $\mathcal{M}_{a}$ to $E^{\prime}$. Then $M\left(G^{\prime}\right)$ is the intersection of the matroids $\mathcal{M}_{1}^{\prime}, \ldots, \mathcal{M}_{m}^{\prime}$ on $E^{\prime}$.

As a consequence of this lemma, the number of matroids needed to represent the matchings in the complete graph $K_{n}$ on $n$ vertices is a natural upper bound for the number of matroids needed to represent the matching independence system of any graph on at most $n$ vertices, i.e., $\mu(n)=\mu\left(K_{n}\right)$.

\subsection{Matchings as the Intersection of Two Matroids}

We present a complete characterization of the graphs for which the set of matchings is the intersection of at most two matroids, by generalizing the concept of bipartite graphs.

For a bipartite graph $G=\left(V_{1} \times V_{2}, E\right)$, the set of matchings $M(G)$ is the intersection of two (partition) matroids. More generally, we get the following.

Theorem 3 Let $G=(V, E)$ be an m-partite graph. Then $M(G)$ is the intersection of at most $m$ matroids on $E$.

Proof: Let $V_{1} \times \ldots \times V_{m}$ be an $m$-partition of $G$. Use the partition matroids

$$
\mathcal{M}_{i}:=\left\{J \subseteq E:|J \cap \delta(v)| \leq 1 \text { for all } v \in V_{i}\right\}, \quad i=1, \ldots, m .
$$


As we will see in Section $[5$ this upper bound has quite a bit of slack for large $\mu(G)$. Moreover, there are non-bipartite graphs $G$ with $\mu(G)=2$, as can be seen from the following characterization.

Theorem 4 The set of matchings $M(G)$ of a graph $G=(V, E)$ is the intersection of two matroids if and only if $G$ contains no odd cycle of cardinality $\geq 5$ and each triangle of $G$ has at most one vertex with degree $>2$.

Proof: a) Let $G$ be a graph that contains no odd cycle of cardinality $\geq 5$ and all triangles of $G$ have at most one vertex with degree $>2$. We call a triangle isolated if all its vertices have degree 2 . Let $G^{\prime}$ be the graph that we obtain from $G$ by contracting all isolated triangles and by deleting from any other triangle the edge that connects the two vertices of degree 2. Then $G^{\prime}$ is a bipartite graph with bipartition $V_{1}^{\prime} \times V_{2}^{\prime}$. For $a=1,2$, let $V_{a}$ consist of all vertices $v \in V$ that correspond to vertices of $V_{a}^{\prime}$ in $G^{\prime}$. Let $\mathcal{C}_{a}$ consist of all circuits $i^{j k}$ of $M(G)$ such that $i \in V_{a}$ or $\{i, j, k\}$ is a triangle of $G$ and $j, k \in V_{a}$. Then $\mathcal{C}_{a}$ is the circuit system of the matroid $\mathcal{M}_{a}:=\left\{J \subseteq E \mid C \nsubseteq J\right.$ for all $\left.C \in \mathcal{C}_{a}\right\}$ and $M(G)$ is the intersection of $\mathcal{M}_{1}$ and $\mathcal{M}_{2}$.

b) Let $M(G)$ be the intersection of two matroids $\mathcal{M}_{1}$ and $\mathcal{M}_{2}$ on $E$. Suppose $G$ contains an odd cycle $v_{1}, v_{2}, \ldots, v_{2 k+1}, k \geq 2$. Then $\left\{v_{1} v_{2}, v_{2} v_{3}\right\},\left\{v_{2} v_{3}, v_{3} v_{4}\right\}$, $\ldots,\left\{v_{2 k+1} v_{1}, v_{1} v_{2}\right\}$ are circuits of $M(G)$ and hence circuits in at least one of the matroids. W.l.o.g. we obtain that $\left\{v_{1} v_{2}, v_{2} v_{3}\right\}$ and $\left\{v_{2} v_{3}, v_{3} v_{4}\right\}$ are circuits of $\mathcal{M}_{1}$, in contradiction to $\left\{v_{1} v_{2}, v_{3} v_{4}\right\}$ being a matching, see Proposition 11 Suppose $G$ contains a triangle $\{u, v, w\}$ with two additional edges $u z$ and $v z^{\prime}$ (possibly $z=z^{\prime}$ ). There are three $u$-circuits $\{u v, u w\},\{u v, u z\}$, and $\{u w, u z\}$. W.l.o.g. all three are circuits of $\mathcal{M}_{1}$. Because $\{v w, u z\}$ is a matching it follows that $\{u w, v w\},\{u v, v w\}$ are circuits of $\mathcal{M}_{2}$ and hence also $\{u v, u w\}$. But because $\left\{u w, v z^{\prime}\right\}$ is a matching, $\left\{u v, v z^{\prime}\right\}$ cannot be a circuit in any of the two matroids, a contradiction. Consequently, $G$ contains no odd cycle of cardinality $\geq 5$ and each triangle of $G$ has at most one vertex with degree $>2$.

\subsection{IP-Formulation}

Next we describe a characterization of the problem whether the set of matchings in a graph $G=(V, E)$ on $n$ vertices can be represented as the intersection of at most $m$ matroids in terms of necessary and sufficient conditions. This characterization leads in a natural way to an IP-formulation of the introduced problem, which can be solved by standard IP-solvers, for at least not too large values of $n$ and $m$.

Suppose first that the set of matchings $M(G)$ of $G$ is the intersection of $m$ matroids $\mathcal{M}_{1}, \mathcal{M}_{2}, \ldots, \mathcal{M}_{m}$ on $E$. Any matching of $G$ must be independent in each of these matroids and any circuit of $M(G)$ must be dependent (and hence a circuit) in at least one of these matroids. For any matroid $\mathcal{M}_{a}$ and any circuit $\{i j, i k\}$ of $M(G)$ with $j<k$, we introduce a $0 / 1$-variable $x_{i j, i k}^{a}$ which is 1 if the 
circuit $\{i j, i k\}$ is dependent (and hence a circuit) in $\mathcal{M}_{a}$ or 0 otherwise, i.e.,

$$
x_{i j, i k}^{a}=\left\{\begin{array}{lll}
1 & : & \{i j, i k\} \text { is dependent in } \mathcal{M}_{a} \\
0 & : & \text { otherwise }
\end{array}\right.
$$

Cover condition. Because any circuit is dependent in at least one of the matroids, we obtain the following cover-inequalities

$$
\sum_{a=1}^{m} x_{i j, i k}^{a} \geq 1 \quad \text { for all } i j, i k \in E, j<k .
$$

Claw condition. For any $i, j, k, l$ different, it is not possible that exactly two of the three circuits $\{i j, i k\},\{i j, i l\}$, and $\{i k, i l\}$ of $M(G)$ are circuits in the same matroid $\mathcal{M}_{a}$, i.e., we have

$$
x_{i j, i k}^{a}+x_{i j, i l}^{a}+x_{i k, i l}^{a} \neq 2 \text { for all } i j, i k, i l \in E, j<k<l \text {, for all } a .
$$

This is modeled by the following claw-inequalities:

$$
\left.\begin{array}{l}
+x_{i j, i k}^{a}+x_{i j, i l}^{a}-x_{i k, i l}^{a} \leq 1 \\
+x_{i j, i k}^{a}-x_{i j, i l}^{a}+x_{i k, i l}^{a} \leq 1 \\
-x_{i j, i k}^{a}+x_{i j, i l}^{a}+x_{i k, i l}^{a} \leq 1
\end{array}\right\} \begin{aligned}
& \text { for all } i j, i k, i l \in E, \\
& j<k<l, \\
& \text { for all } a
\end{aligned}
$$

Triangle condition. For any $i j, i k, j k \in E$ different, it is impossible that exactly two of the three circuits $\{i j, i k\},\{j i, j k\}$, and $\{k i, k j\}$ of $M(G)$ are circuits in the same matroid $\mathcal{M}_{a}$, i.e., we have

$$
x_{i j, i k}^{a}+x_{j i, j k}^{a}+x_{k i, k j}^{a} \neq 2 \text { for all } i j, i k, j k \in E, i<j<k \text {, for all } a .
$$

We obtain the triangle-inequalities:

$$
\left.\begin{array}{l}
+x_{i j, i k}^{a}+x_{j i, j k}^{a}-x_{k i, k j}^{a} \leq 1 \\
+x_{i j, i k}^{a}-x_{j i, j k}^{a}+x_{k i, k j}^{a} \leq 1 \\
-x_{i j, i k}^{a}+x_{j i, j k}^{a}+x_{k i, k j}^{a} \leq 1
\end{array}\right\} \begin{aligned}
& \text { for all } i j, i k, j k \in E \\
& i<j<k \\
& \text { for all } a
\end{aligned}
$$

Matching condition. For any $i, j, k, l$ different with $i j, k l \in E,\{i j, k l\}$ is a matching in $G$. Hence, it is not possible that both circuits $\{i j, i k\}$ and $\{k i, k l\}$ of $M(G)$ are circuits in the same matroid $\mathcal{M}_{a}$. This leads us to the matching-inequalities:

$$
\begin{aligned}
& x_{i j, i k}^{a}+x_{k i, k l}^{a} \leq 1 \text { for all } i j, i k, k l \in E, j<k, i<l, j \neq l, \text { for all } a \\
& x_{i k, i j}^{a}+x_{k i, k l}^{a} \leq 1 \text { for all } i j, i k, k l \in E, k<j, i<l, j \neq l, \text { for all } a \\
& x_{i k, i j}^{a}+x_{k l, k i}^{a} \leq 1 \text { for all } i j, i k, k l \in E, k<j, l<i, j \neq l, \text { for all } a
\end{aligned}
$$

Theorem 5 The set of matchings $M(G)$ of a graph $G$ is the intersection of at most $m$ matroids if and only if there exists $x_{i j, i k}^{a} \in\{0,1\}$ for all $i j, i k \in E, j<k$, for all $a=1, \ldots, m$ such that all the inequalities in (1), (2), (3), (4) are satisfied. 
Proof: We have to show that any feasible solution of the given system leads to $m$ matroids such that $M(G)$ is their intersection. Let $\left(x_{i j, i k}^{a}\right)_{i, j, k, a}$ be such a feasible solution. For $a=1,2, \ldots, m$, define

$$
\mathcal{C}_{a}:=\left\{\{i j, i k\}: i j, i k \in E, j<k, x_{i j, i k}^{a}=1\right\} .
$$

Due to the claw-, triangle-, and matching-inequalities, $\mathcal{C}_{a}$ is the circuit system of a matroid. Its associated matroid is $\mathcal{M}_{a}=\left\{J \subseteq E: C \not \subseteq J\right.$ for all $\left.C \in \mathcal{C}_{a}\right\}$. We claim that $M(G)$ is the intersection of $\mathcal{M}_{1}, \mathcal{M}_{2}, \ldots, \mathcal{M}_{m}$.

Let $J \in M(G)$. Because any circuit of $\mathcal{M}_{a}$ is a circuit of $M(G), J$ is an element of $\mathcal{M}_{1} \cap \cdots \cap \mathcal{M}_{m}$. Now let $J$ be an element of this intersection. Suppose $J$ is not in $M(G)$. Then there is some circuit $\{i j, i k\}(j<k)$ in $M(G)$ which is contained in $J$. Due to the cover-inequalities, $\{i j, i k\}$ is a circuit in at least one matroid $\mathcal{M}_{a}$, in contradiction to $J$ being $\mathcal{M}_{a}$-independent.

For most IP-solvers it is more efficient to solve an optimization problem instead of solving a feasibility problem. We transform the feasibility problem into an optimization problem by introducing additional $0 / 1$-variables $y_{i j, i k}$ for any circuit $\{i j, i k\}(j<k)$ of $M(G)$. We replace the cover-inequalities (11) by the inequalities

$$
\sum_{a=1}^{m} x_{i j, i k}^{a}-y_{i j, i k} \geq 0 \quad \text { for all } i j, i k \in E, j<k
$$

and try to maximize the sum of the new $y$-variables, i.e., $\max \sum_{i j, i k \in E, j<k} y_{i j, i k}$. This means we want to cover as many circuits as possible. Consequently, the original feasibility problem has a feasible solution if and only if the new program has a feasible solution in which all $y$-variables are equal to 1 . Note that the 0 vector is a feasible starting solution for this integer program.

Nevertheless, these problems are still hard to solve. The integer programs that we explore are quite large and grow very fast because the problems have $\mathcal{O}\left(m n^{3}\right)$ variables and $\mathcal{O}\left(m n^{4}\right)$ constraints. Therefore, current IP-solvers, e.g., $\mathrm{CPLEX}^{1}$ or $\mathrm{SIP}^{2}$, are unable to handle them in reasonable time even for moderate values of $n$ and $m$ (e.g., $G=K_{n}$ with $n=13, m=4$ ).

\section{Lower Bounds}

In the following, we use the notation $\nu(m)$ to indicate the largest $n$ for which $\mu(n) \leq m$, i.e.,

$$
\nu(m)=\sup \{n \in \mathbb{N}: \mu(n) \leq m\} .
$$

The following result shows that $\nu(m)$ is indeed finite and grows at most doubly exponentially.

\footnotetext{
${ }^{1}$ CPLEX Linear Optimizer 6.0 (with Mixed Integer \& Barrier Solvers); (C) ILOG Inc., Incline Village, NV, USA.

${ }^{2}$ Solving Integer Programs 1.1 by Alexander Martin (TU Darmstadt, Germany), unpublished.
} 
Theorem $6 \nu(m) \leq 2^{2^{3 m}-1}-1$.

Proof: Let $G=(V, E)$ be the complete graph $K_{n}$ on $n$ vertices and $\mu(n) \leq$ $m$. We start by introducing some technical terms. For a vertex $i$, consider all directed edges $i j, j \in V \backslash\{i\}$. (The difference between $i j$ and $j i$ is only important for the following definition.) If an $i$-circuit involving edge $i j$ is covered by matroid $\mathcal{M}_{a}$, we say that ij has color $a$. The set of colors of an edge defines its color type. The color class of any vertex $i$ is the set of color types of all the edges $i j, j \in V \backslash\{i\}$. Finally, a degenerate triangle consists of three different vertices $i, j, k$, such that all three circuits $\{i j, i k\},\{j i, j k\},\{k i, k j\}$ are covered by the same matroid, say, $\mathcal{M}_{a}$. For such a triangle, it is an easy consequence of the matching condition described in Section 3.2 that none of the other circuits involving the edges $i j, i k, j k, j i, k i, k j$ may be covered by $\mathcal{M}_{a}$; i.e., for $l \notin\{i, j, k\}$, none of the circuits $\{i j, i l\},\{i k, i l\},\{j i, j l\},\{j k, j l\},\{k i, k l\}$, $\{k j, k l\}$ may be covered by $\mathcal{M}_{a}$ as well.

We start by eliminating covers with degenerate triangles. If there are any degenerate triangles in a circuit cover with $m$ matroids, we can construct a circuit cover with $3 m$ matroids that is free of degenerate triangles: For each degenerate triangle covered by matroid $\mathcal{M}_{a}$, cover one circuit by matroid $\mathcal{M}_{a}$, the other two by the additional matroids $\mathcal{M}_{a^{\prime}}$ and $\mathcal{M}_{a^{\prime \prime}}$. By the observation at the end of the preceding paragraph, this does not affect any other circuits already covered by $\mathcal{M}_{a}$, so all conditions described in Section 3.2 are still valid. Therefore, this yields indeed a feasible set of matroids.

Now consider the situation in the absence of degenerate triangles. As any (directed) edge $i j \in E$ is part of some circuit, and each circuit is covered by some matroid, there can be at most $2^{m}-1$ different color types for $m$ different matroids. Moreover, there are at most $2^{2^{m}-1}-1$ different color classes of vertices. Furthermore, any $i$-circuit formed by a pair of edges $i j, i k$ with $j \neq k$ must be covered by some matroid, so no two color types in any valid color class can be disjoint.

Assume that we have two vertices $(i$ and $j$ ) of the same color class. Then their connecting edge $i j$ is part of a circuit $\{i j, i k\}$ that is covered by some matroid $\mathcal{M}_{a}$; at the same time, there must be a circuit $\{j i, j l\}$ that is also covered by $\mathcal{M}_{a}$. If $k \neq l$, we get a violated matching condition. If $k=l$, we use the fact that $\{i, j, k\}$ is not a degenerate triangle, and we conclude that we get a violated triangle condition.

Therefore, there can be at most $2^{2^{m}-1}-1$ vertices in the absence of degenerate triangles, and not more than $2^{2^{3 m}-1}-1$ in general.

Quite clearly, upper bounds for $\nu(m)$ correspond to lower bounds for $\mu(n)$. In particular, we get

Corollary $7 \mu(n) \in \Omega(\log \log n)$. 


\section{Upper Bounds}

Using a recursive construction, we can show that $m$ matroids suffice to generate the matchings of any graph with $O(\sqrt[3]{m !})=2^{O(m \log m)}$ vertices. As above, this yields an upper bound for $\mu(n)$. More precisely, we show

\section{Theorem 8}

$$
\nu(m) \geq 3 \prod_{i=0}^{\left\lceil\frac{m}{3}\right\rceil-2}(m-3 i) .
$$

Proof: We proceed by induction. Clearly, the claim is true for $m=1,2,3$, as all three circuits of $K_{3}$ can be covered by the circuit system of just one matroid. Now suppose the claim was true for $m-3$. Consider $m$ vertex sets $V_{1}, \ldots, V_{m}$, each consisting of $\nu(m-3)$ vertices.

For proving the overall claim, it suffices to describe circuit systems $\mathcal{C}_{1}, \ldots, \mathcal{C}_{m}$ that satisfy the following conditions.

(1) All circuits $v^{u w}$ with $v, u, w \in V_{k}$ are contained in some $\mathcal{C}_{i}$.

(2) All circuits $v^{u w}$ with $v \in V_{k}, u, w \notin V_{k}$ are contained in some $\mathcal{C}_{i}$.

(3) All circuits $v^{u w}$ with $v, u \in V_{k}, w \notin V_{k}$ are contained in some $\mathcal{C}_{i}$.

(4) Each resulting $\mathcal{C}_{i}$ is the circuit system of a matroid.

In the following, indices are taken modulo $m$. By the induction hypothesis, the set of matchings on $V_{k}$ is the intersection of $m-3$ matroids. Denote the corresponding circuit systems by $\mathcal{C}_{1}^{k}, \ldots, \mathcal{C}_{m-3}^{k}$. For $i=1, \ldots, m$ define

$$
\mathcal{C}_{i}^{\prime}:=\mathcal{C}_{1}^{i+2} \cup \mathcal{C}_{2}^{i+3} \cup \ldots \cup \mathcal{C}_{m-3}^{i-2}=\bigcup_{j=1}^{m-3} \mathcal{C}_{j}^{i+1+j} .
$$

In particular, $\mathcal{C}_{k-i-1}^{k} \subseteq \mathcal{C}_{i}^{\prime}$ is the set of all circuits within $V_{k}$ that are covered by $C_{i}^{\prime}$. Then $\mathcal{C}_{i}^{\prime}$ is a circuit system on $E$ and $\mathcal{C}_{1}^{\prime}, \ldots, \mathcal{C}_{m}^{\prime}$ satisfy condition (1). The circuit systems $\mathcal{C}_{1}, \ldots, \mathcal{C}_{m}$ arise now by adding $\mathcal{C}_{i}^{\prime}$ to $\mathcal{C}_{i}$ and for $k=1, \ldots, m$ :

- Add $\left\{v^{u w}: v \in V_{k}, u, w \notin V_{k}\right\}$ to $\mathcal{C}_{k}$.

Add $\left\{v^{u w}: v \in V_{k}, u, w \in V_{k+1}\right\}$ to $\mathcal{C}_{k-1}$.

Add $\left\{v^{u w}: v \in V_{k}, u, w \notin V_{k} \cup V_{k+1}\right\}$ to $\mathcal{C}_{k+1}$.

- Now consider $V_{k}=\left\{v_{(k-1) \nu(m-3)+1}, \ldots, v_{k \nu(m-3)}\right\}$.

Add the following circuits to $\mathcal{C}_{k}$

$$
\left\{v_{i}^{v_{j} w}: i<j, v^{i}, v^{j} \in V_{k}, w \notin V_{k}\right\} \cup\left\{v_{i}^{v_{j} v_{l}}: i<j, l, v^{i}, v^{j}, v^{l} \in V_{k}\right\} .
$$

Add the following circuits to $\mathcal{C}_{k-1}$

$$
\left\{v_{i}^{v_{j} w}: i>j, v^{i}, v^{j} \in V_{k}, w \in V_{k+1}\right\} \cup\left\{v_{i}^{v_{j} v_{l}}: i>j, l, v^{i}, v^{j}, v^{l} \in V_{k}\right\} .
$$

Add the following circuits to $\mathcal{C}_{k+1}$

$$
\left\{v_{i}^{v_{j} w}: i>j, v^{i}, v^{j} \in V_{k}, w \notin V_{k} \cup V_{k+1}\right\} \cup\left\{v_{i}^{v_{j} v_{l}}: i>j, l, v^{i}, v^{j}, v^{l} \in V_{k}\right\} .
$$




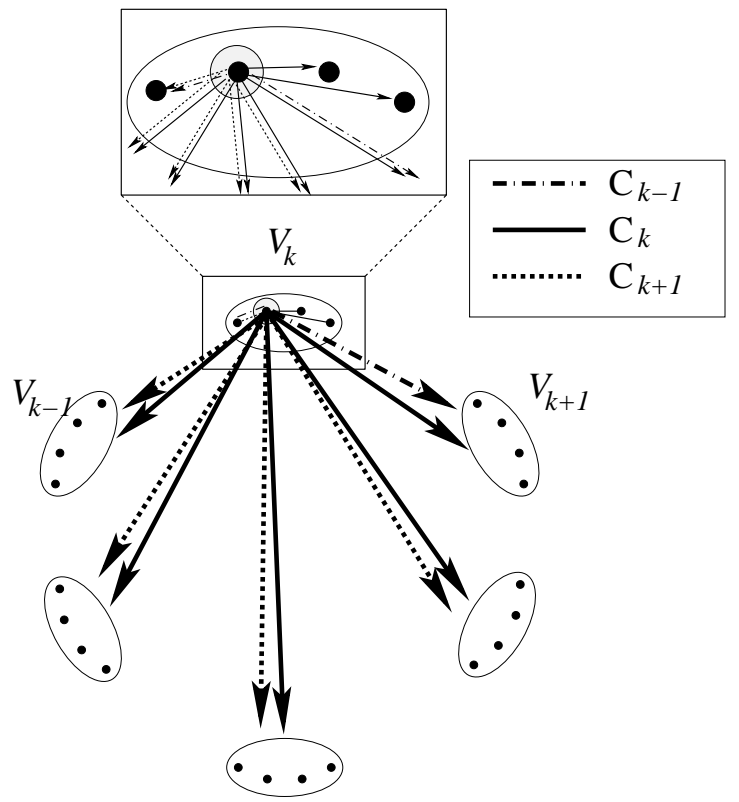

Figure 1: The structure of those circuits in $\mathcal{C}_{k-1}, \mathcal{C}_{k}, \mathcal{C}_{k+1}$ that have a central vertex in $V_{k}$.

See Fig. 1 for an illustration. There, arrowheads point away from the central vertices of circuits.

The resulting circuit systems $\mathcal{C}_{1}, \ldots, \mathcal{C}_{m}$ satisfy conditions (1), (2), and (3). In order to see that each $\mathcal{C}_{i}$ is the circuit system of a matroid, we need to verify the claw-, triangle-, and matching-conditions described in Section 3.2

Claw condition: We need to verify that for any two circuits $v^{u w_{1}}, v^{u w_{2}}$ that are both contained in some $\mathcal{C}_{i}, v^{w_{1} w_{2}}$ is also contained in $\mathcal{C}_{i}$.

Let $v \in V_{k}$. If $i \notin\{k-1, k, k+1\}$, then the involved circuits must be completely within $V_{k}$ and they are all covered by $\mathcal{C}_{k-i-1}^{k} \subseteq \mathcal{C}_{i}$. Otherwise, $i \in\{k-1, k, k+1\}$ and the property is easily verified.

Triangle and matching conditions: Let $v^{u w_{1}} \in \mathcal{C}_{i}$ for some $i$. We need to verify that if $u^{v w_{2}} \in \mathcal{C}_{i}$ for some $w_{2}$ then $w_{2}=w_{1}$ and $w_{1}^{u v} \in \mathcal{C}_{i}$.

Let $v \in V_{k}$. If $i \notin\{k-1, k, k+1\}$, then the involved circuits must be completely within $V_{k}$ and hence, being in $\mathcal{C}_{i}$ means being in $\mathcal{C}_{k-i-1}^{k}$. Because $\mathcal{C}_{k-i-1}^{k}$ is the circuit system of a matroid, the triangle and matching conditions are satisfied. In the following $i \in\{k-1, k, k+1\}$. It suffices to show that no $u^{v w_{2}}$ is contained in $\mathcal{C}_{i}$. If $u \notin V_{k} \cup V_{k+1}$ then $i=k$ or $i=k+1$. On the other hand, none of the circuits $u^{v w_{2}}$ is in $\mathcal{C}_{k}$ or $\mathcal{C}_{k+1}$. If $u \in V_{k+1}$ it follows by our construction that $i=k$ or $i=k-1$ while $u^{v w_{2}}$ is in $\mathcal{C}_{k+2}$ or $\mathcal{C}_{k+1}$. Finally, we consider $u \in V_{k}$. Let $v=v_{h}$ and $u=v_{j}$. If $h<j$ then $i=k$ while $u^{v w_{2}}$ is in $\mathcal{C}_{k-1}$ or $\mathcal{C}_{k+1}$. If $h>j$ then $i=k-1$ or $i=k+1$ while $u^{v w_{2}}$ is in $\mathcal{C}_{k}$. 
Consequently, $\mathcal{C}_{1}, \ldots, \mathcal{C}_{m}$ are circuit systems of matroids, say $\mathcal{M}_{1}, \ldots, \mathcal{M}_{m}$, and all circuits of $M(G)$ are covered by some $\mathcal{C}_{i}$. Hence, $M(G)$ is the intersection of the matroids $\mathcal{M}_{1}, \ldots, \mathcal{M}_{m}$. Therefore, $\nu(m) \geq m \cdot \nu(m-3)$. With $\mu(j) \geq 3$ for $j \in\{1,2,3\}$ we obtain

$$
\nu(m) \geq 3 \prod_{i=0}^{\left\lceil\frac{m}{3}\right\rceil-2}(m-3 i) .
$$

Now the lower bound on $\nu(m)$ implies an upper bound on $\mu(n)$ :

Corollary $9 \mu(n) \in O(\log n / \log \log n)$.

Proof: For $n \geq s=2^{c m \log m}$, i.e., $m \log m=\frac{\log s}{c}$, we get $\log m+\log \log m=$ $\log (m \log m)=\log \log s-\log c$. This implies $2 \log m \geq \log \log s$ for sufficiently large $m$. Therefore, $\frac{2 \log n}{c \log \log n} \geq \frac{2 \log s}{c \log \log s} \geq m$.

\section{Tight Bounds for $\mu(n)$}

Lawler mentioned in 12] that the nonbipartite matching problem can be formulated as an intersection problem involving two partition matroids, but with additional constraints in the form of symmetry conditions. Nevertheless, we can give an elementary proof of the following.

Theorem $10 \mu(n)=4$ for $n=5, \ldots, 12$.

Proof: From Theorem 8 we obtain $\nu(4) \geq 12$, hence, $\mu(n) \leq 4$ for $n \leq 12$.

We complete the proof by showing that $\mu(5) \geq 4$. Let $G$ be the complete graph $K_{5}$ with vertex set $\{1,2,3,4,5\}$ and $M(G)$ the set of matchings of $G$. Suppose $M(G)$ is the intersection of three matroids $\mathcal{M}_{1}, \mathcal{M}_{2}$, and $\mathcal{M}_{3}$ on $E$.

Consider the circuits that correspond to one vertex with incident edges $a, b, c, d$. These are $\{a, b\},\{a, c\},\{a, d\},\{b, c\},\{b, d\},\{c, d\}$.

Suppose exactly three of them are $\mathcal{M}_{1}$-circuits, w.l.o.g., $\{a, b\},\{a, c\},\{b, c\}$. Without loss of generality, $\{a, d\}$ and $\{b, d\}$ and hence $\{a, b\}$ are $\mathcal{M}_{2}$-circuits. There exist edges $e$ and $f$ such that $\{a, b, e\}$ and $\{b, d, f\}$ are triangles:

Because $\{c, e\}$ is a matching $\{a, e\}$ is no $\mathcal{M}_{1}$-circuit and because $\{d, e\}$ is a matching $\{a, e\}$ is no $\mathcal{M}_{2}$-circuit, hence, $\{a, e\}$ is an $\mathcal{M}_{3}$-circuit, Similarly we obtain $\{b, e\}$ is an $\mathcal{M}_{3}$-circuit. Consequently, $\{a, b\}$ is an $\mathcal{M}_{1^{-}}$, an $\mathcal{M}_{2^{-}}$, and an $\mathcal{M}_{3}$-circuit. Because $\{a, f\}$ is a matching, there is no possibility for $\{b, f\}$ to be a circuit in one of the three matroids, a contradiction.

Hence, it is not possible that any matroid has exactly three of the six circuits that correspond to one vertex as circuits. If a matroid has at least four of the six circuits that correspond to one vertex as circuits then all six circuits are its circuits. Therefore, all six circuits are circuits in the same matroid or each of the three matroids has exactly two of the six circuits as circuits, these must be disjoint. Consequently, there are $i_{1}, i_{2}, i_{3}, i_{4}, i_{5} \in\{1,2,3\}$ such that $\{12,15\}$ and 
$\{13,14\}$ are $\mathcal{M}_{i_{1}}$-circuits, $\{12,23\}$ and $\{24,25\}$ are $\mathcal{M}_{i_{2}}$-circuits, $\{23,34\}$ and $\{13,35\}$ are $\mathcal{M}_{i_{3}}$-circuits, $\{34,45\}$ and $\{14,24\}$ are $\mathcal{M}_{i_{4}}$-circuits, and $\{45,15\}$ and $\{25,35\}$ are $\mathcal{M}_{i_{5}}$-circuits. Up to symmetry, we obtain $i_{1}=i_{2}$, i.e., $\{12,15\}$, $\{13,14\},\{12,23\}$, and $\{24,25\}$ are $\mathcal{M}_{i_{1}}$-circuits, in contradiction to $\{15,23\}$ being a matching.

We can also give a positive result concerning matching and the intersection of three matroids:

Theorem 11 Let $G=(V, E)$ be a 4-partite graph. Then the set of matchings $M(G)$ of $G$ is the intersection of at most three matroids on $E$.

Proof: Let $V_{1} \times V_{2} \times V_{3} \times V_{4}$ be a 4-partition of $G$. For $a=1,2,3$, let $\mathcal{C}_{a}$ consist of all $V_{a}$-circuits and of all $V_{4}$-circuits $i^{j k}$ with $j, k \notin V_{a}\left(i \in V_{4}\right)$. Then $\mathcal{C}_{a}$ is the circuit system of a matroid. Its associated matroid is $\mathcal{M}_{a}=\{J \subseteq E$ : $C \nsubseteq J$ for all $\left.C \in \mathcal{C}_{a}\right\}$. It is easy to see that $M(G)$ is the intersection of the matroids $\mathcal{M}_{1}, \mathcal{M}_{2}, \mathcal{M}_{3}$ on $E$.

This implies that the set of matchings of any subgraph of $K_{5}$ is the intersection of at most three matroids, i.e., $K_{5}$ is the smallest graph for which the set of matchings is not the intersection of three matroids.

Theorem 8 implies that $\nu(5) \geq 15$, hence, $\mu(n) \leq 5$ for $n=13,14,15$. Using refined versions of the techniques for the lower bound described in Section 4 we can show the following:

Theorem $12 \mu(n)=5$ for $n=13,14,15$.

The proof proceeds by showing that for $m=4$, there are at most 12 "basic" color classes, i.e., color classes that cannot be simplified by deleting some of the circuits from some of the matroids. This implies the claim in the absence of degenerate triangles, as there cannot be any two vertices from the same basic color class. Furthermore, any pair of vertices from the same basic color class (which forces a degenerate triangle) eliminates another basic color class; again, the claim follows.

As full details are rather tedious and probably not of sufficient interest to the reader, they are omitted.

\section{References}

[1] W. J. Cook, W. H. Cunningham, W. R. Pulleyblank, and A. Schrijver. Combinatorial Optimization. Wiley-Interscience, New York, 1998.

[2] J. Edmonds. Paths, trees, and flowers. Canadian Journal of Mathematics, 17 (1965), pp. 449-467.

[3] J. Edmonds. Submodular functions, matroids and certain polyhedra. In: Combinatorial Structures and their Applications (R. K. Guy, H. Hanai, N. Sauer, and J. Schönheim, eds.) Gordon and Brach, New York (1970), pp. 69-87. 
[4] J. Edmonds. Matroid Intersection. Annals of Discrete Mathematics, 4 (1979), pp. 39-49.

[5] S. P. Fekete, R. T. Firla, and B. Spille. Matching as the Intersection of Matroids. Electronic Notes in Discrete Mathematics, Volume 10 (2001).

[6] A. Frank. A weighted matroid intersection algorithm. Journal of Algorithms, 2 (1981), pp. 328-336.

[7] R. L. Graham, M. Grötschel, and L. Lovász (eds.) Handbook of combinatorics. Volume 1-2. Elsevier (North-Holland), Amsterdam, 1995.

[8] M. Grötschel, L. Lovász, and A. Schrijver. Geometric algorithms and combinatorial optimization. Springer, Berlin, 1988.

[9] T. Jenkyns. The efficacy of the greedy algorithm. Proc. 7th Southeastern Conference on Combinatorics, Graph Theory, and Computation (1976), pp. 341-350.

[10] B. Korte and D. Hausmann. Lower bounds on the worst-case complexity of some oracle algorithms. Discrete Mathematics 24 (1978), pp. 261-276.

[11] E. L. Lawler. Matroid intersection algorithms. Mathematical Programming, 9 (1975), pp. 31-56.

[12] E. L. Lawler. Combinatorial Optimization: Networks and Matroids. Holt, Rinehart and Winston, New York, 1976.

[13] J. G. Oxley. Matroid Theory. Oxford University Press, Oxford, 1992.

[14] D. J. A. Welsh. Matroids: Fundamental concepts. In: Handbook of Combinatorics, Volume 1-2, (R. L. Graham, M. Grötschel, and L. Lovász, eds.) Elsevier (North-Holland), Amsterdam, 1995, pp. 481-526. 